\title{
Application study of a correction method for a spacecraft thermal model with a Monte-Carlo hybrid algorithm
}

\author{
CHENG WenLong ${ }^{1 *}$, LIU Na $^{1}$, LI Zhi $^{2}$, ZHONG Qi $^{3}$, WANG AiMing ${ }^{2}$, ZHANG ZhiMin $^{2}$ \& \\ HE ZongBo ${ }^{2}$
}

${ }^{1}$ Department of Thermal Science and Energy Engineering, University of Science and Technology of China, Hefei 230027, China;

${ }^{2}$ Research and Development Center, China Academy of Space Technology, Beijing 100094, China;

${ }^{3}$ Beijing Institute of Spacecraft System Engineering, Chinese Academy of Space Technology, Beijing 100094, China

Received January 12, 2010; accepted April 6, 2010

\begin{abstract}
The correction of a thermal model for a thermally controlled satellite in ground test conditions is studied using a Monte Carlo hybrid algorithm. First, the global and local parameters are summarized according to sensitivity analyses on uncertain parameters, and then the model correction is treated as a parameter optimization problem to be solved with a hybrid algorithm. Finally, the correction of the thermal model is completed using a layered correction method. The sensitivity analysis showed that the effective emissivities across the multi-layer insulation (MLI) and the emissivities of the thermal control coating are global parameters, while the contact heat transfer coefficients are local parameters. After correction, the deviations between the calculated and test values were all within $\pm 3^{\circ} \mathrm{C}$. The final results prove that the method in this study is superior to traditional methods and satisfies the requirements for thermal model correction.
\end{abstract}

model correction, thermal model, thermal balance test results, Monte Carlo

Citation: Cheng W L, Liu N, Li Z, et al. Application study of a correction method for a spacecraft thermal model with a Monte-Carlo hybrid algorithm. Chinese Sci Bull, 2011, 56: 1407-1412, doi: 10.1007/s11434-010-4053-z

Due to the complexity of an actual spacecraft, its thermal analysis network model generally requires simplifications and reasonable assumptions. At the same time, the input parameters of the thermal model, such as the contact heat transfer coefficients, thermal optical properties of surface coating and so on, are subject to uncertainty. The combination of these factors leads to deviations between results from the thermal network method and spacecraft thermal balance test or telemetry system. Sometimes these deviations are relatively large. To minimize calculation deviations and improve the ability of the model to achieve more accurate spacecraft temperature analysis, it is essential to correct the thermal model [1].

Currently the commonly used correction methods are the least-squares method correcting the integrated radiation

*Corresponding author (email: wlcheng@ustc.edu.cn) factors [2] and the semi-empirical trial method [3]. However, the accuracy of the thermally corrected model largely depends on empirical judgments of analysts in the above methods, so there may be large errors in the correction of some key parameters. In addition, with the increasing complexity of the spacecraft's internal structure and layout, there are increasing uncertainties in the thermal analysis. Because of the increasingly uncertain parameters and the correlation or coupling between them, corrections by traditional methods not only result in a heavy workload, but also cannot satisfy the progressively higher temperature control requirements. Therefore, improvement of the correction method is very necessary.

The Monte-Carlo method has been widely used in structural analysis, radiation and mass flux calculation, geophysical inversion studies, measurement of thermophysical properties, satellite detection of atmospheric ozone and 
many other areas [4-10] because of its simple principles and universally-wide applicability. The method is more suitable to the correction of multi-parameter and large-scale thermal models than traditional methods ${ }^{1)}$, so the application prospects of the method in the field of space thermal model correction has attracted considerable attention from scholars, both in China and abroad, in recent years ${ }^{2}$, $[11,12]$. Mareschi et al. [13] used the method to improve the efficiency of the correction of the INTEGRAL satellite thermal model, but its accuracy needs to be improved. Cheng et al. $[14,15]$ corrected a virtual satellite thermal model and obtained good effect when the optical properties of spacecraft surface coatings, contact heat transfer coefficients and so on were regarded as uncertain parameters. However, only a virtual satellite was used in the two references, and owing to the lack of experimental data, the calculated results of the thermal model were treated as "experimental values" in the correction. Therefore, in the thermal model correction, the "true values" of uncertain parameters were known. In fact, the true values of uncertain parameters of a real satellite are unknown over a wide range, so the model correction is more difficult. In addition, the effect of the multi-layer insulation (MLI) thermal parameters on the spacecraft temperatures was not considered in the model.

Research work on spacecraft thermal model correction based on the Monte-Carlo method has only just begun in China, and has so far been generally about what effects will be produced when the method is applied to engineering practice. Moreover, for the specific correction method and the steps to be taken, more in-depth research is needed.

For the above reasons, the thermal model for a thermally controlled satellite in ground test conditions was corrected in accordance with thermal balance test data based on a Monte-Carlo hybrid algorithm. First, the global and local parameters were summarized according to the sensitivity analyses on uncertain parameters, and then the model correction was treated as a parameter optimization problem to be solved with a hybrid algorithm. Finally the correction of the thermal model was completed using the layered correction method. After correction, the deviations between calculated and test values were all within $\pm 3^{\circ} \mathrm{C}$, proving that the method in this study is superior to traditional methods $[1,3,16]$ and satisfies the requirements for thermal model correction.

\section{Physical model}

The satellite used a box-type configuration and board structure. Its platform structure consisted of the docking ring, bottom plate, top plate, $\pm Y$ side plate, $\pm Z$ side plate, $Y$-partition and $Z$-partition. The equipment inside the satellite included most electronic equipment installed on the main floor, $Z$-partition, $Y$-partition and $Y$ side plate.

The thermally controlled satellite did not have the solar arrays, antennas and other equipment installed outside it in the ground test conditions. The thermal balance test was done in a vacuum tank, cooled with liquid nitrogen and coated with black paint to simulate the vacuum, cold and black-background environment. Resistance heating sheets were used to simulate the space external heat flux, and thermistors and thermocouples were used to measure temperature.

\section{Thermal analysis model}

\subsection{Simplification of the physical model}

To ensure calculation accuracy, the following reasonable simplifications are made to facilitate the thermal analysis:

(i) ignore all electrical connectors, cable conductors and installation screws inside the satellite;

(ii) ignore all holes formed by the electrical connectors, conductors and equipment installation screws in the equipment installation board or panel;

(iii) consider most of the electronic equipment and convert them to hexahedrons, cylinders or a combination of the two in accordance with their equivalent radiation area;

(iv) ignore the thermal contact between the honeycomb panels;

(v) consider the impact of contact thermal resistance between electronic equipment and associated fitting surfaces.

\subsection{Principle of node partition}

The principles of node partition are listed below:

(i) consider general electronic equipment as an isothermal body and single node model;

(ii) construct three sheets for a honey comb panel, and set the material, thickness and thermal optical properties for the top and bottom face sheets. A honeycomb core is placed between the top and bottom face sheets. If a MLI is attached to the face sheet, the corresponding outer surface of the MLI is a node and its thickness is zero;

(iii) node partition of honeycomb board with pre-buried heat pipes is in accordance with the distribution of heat pipes, and each heat pipe is divided into at least three nodes.

\subsection{Calculation model}

The thermal model of the thermally controlled satellite was built in accordance with the principles of model simplification and node partition. The powers of the heating sheets on

1) Molina M, Finzi A E. Monte Carlo techniques for thermal analysis of space vehicles: Practical examples of robustness determination in preliminary design. In: AIDAA VIII Congresso Nazionale, 2005

2) Zhong Q, Liu W, Ma H T. Overview of thermal model correction. In: The Seventh Conference on Space Thermal Physics, 2005 
the outer surfaces of the satellite were introduced into the thermal model as the absorbed external heat flux. The thermal model of the thermally controlled satellite is shown in Figure 1 (a).

The thermal balance test was done in a vacuum tank. Therefore, it was necessary to build a thermal model for the vacuum tank. In the modeling, the main simplifications and parameter settings were as follows: (1) the heat sink, guide rail and test bench were considered, but the cables, brackets and other components were ignored; (2) the impact of rarefied gas in the vacuum tank was not considered; and (3) the heat sink temperature was $-190^{\circ} \mathrm{C}$, and the emissivity and absorptivity of the vacuum tank's internal surface coating was 0.90 . The thermal model of the vacuum tank is shown in Figure 1(b).

The thermal model of the thermally controlled satellite was placed in the model of the vacuum tank in accordance with the requirements for the location of the test, and their combination was the calculation model, which is shown in Figure 1(c).

\section{Thermal model corrections}

\subsection{The optimization model and optimization algo- rithm}

In this study, SINDA / FLUINT was used to help correct the thermal model. In essence, it selects uncertain parameters, constructs object functions of the calculated and test data, and solves the parameter optimization problem to achieve the model correction. The object function refers to the errors between the calculated and test data and its expression depends on the data fitting method. In this study, the least square error method was used to construct object function, and the optimization model is as follows:

$$
\min \left(\sqrt{\sum_{i=1}^{N}\left(P_{i}-T_{i}\right)^{2} / N}\right),
$$

with $N$ as the total number of items of equipment, $P_{i}$ the predication of the equipment $i$, and $T_{i}$ the test data for the equipment $i$.
As a single-objective unconstrained optimization problem constructed in eq. (1), it can be solved mainly by the following algorithm: a quasi-linear optimization method, Monte Carlo method and hybrid algorithm [15]. In fact, the hybrid algorithm is an application of the combination of quasi-linear optimization and the Monte Carlo method. Cheng et al. [15] found the hybrid algorithm can obtain higher precision results and consume less calculation time compared with the other two methods. Moreover, its result does not depend on initial values, so it is particularly suitable for problems with less prior information. Therefore, the hybrid algorithm was used in this study.

\subsection{Correction process for thermal model}

The first step in thermal model correction was sensitivity analyses to identify key uncertain parameters. The second step was to select the appropriate correction method to correct the thermal model.

Under these test conditions, the key uncertain parameters affecting the satellite temperatures are mainly the effective emissivity across the MLI, the emissivity of the thermal control coating, and the contact heat transfer coefficient between the equipment and installation board. Sensitivity analyses were made on the uncertain parameters with the Monte-Carlo method [14]. Through the sensitivity analyses we found that the effective emissivities across the MLI and the emissivities of the thermal control coating, defined as key global parameters, have considerable impacts on almost all the satellite equipment, and the contact heat transfer coefficients, defined as key local parameters, only had an impact on equipment associated with the specified contact heat transfer coefficient. Using the layered correction method, the thermal model correction process was done in three steps:

(1) correct the key global parameters taking all parameters as variables;

(2) take the first step's results as the key global parameter values and correct key local parameters;

(3) take the first two steps' parameter correction results as initial values to further correct them with the quasi-linear optimization algorithm, specifically referring to the BFGS

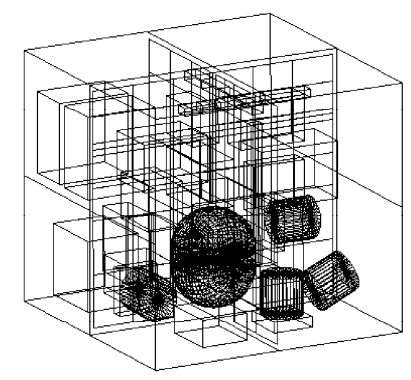

(a)

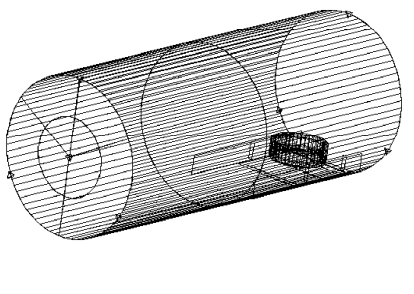

(b)

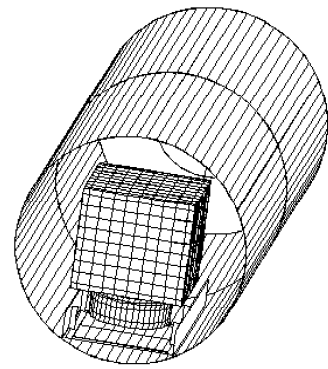

(c)

Figure 1 Thermal network model. (a) Thermal model of a thermally controlled satellite; (b) thermal model of vacuum tank; (c) thermal model in ground test conditions. 
method [17] in this study.

Uncertain parameters are presented in Table 1. The optical properties of thermal control coatings are associated with the coating type and each of the thermal control coatings is interrelated. The contact heat transfer coefficients are related to the equipment. The effective emissivity of each region is independent.

\section{Calculation results and discussion}

The calculation conditions are presented in Table 2. The heat flux in the steady-state conditions 1 and 2 are the same, but active temperature control requirements for the equipment in the two conditions are different. Due to the existence of the shadow zone, the calculation for transient condition 1 was a transient simulation and averages were obtained for the equipment temperatures. Integral average values of the orbital period external thermal flux were used to calculate all the satellite temperatures in the steady-state conditions 1 and 2 .

A group of thermal balance tests in the steady-state condition 1 was carried out, and the results (shown in Figure 2) were taken as test data. Three and two groups of thermal balance tests were done in the transient condition and steady-state condition 2, respectively, and the averages of results in every condition were taken as test data (shown in Figures 3 and 4). The average deviation and standard deviation for the temperature analysis are defined as follows:

$$
\begin{gathered}
\overline{\Delta T}=\frac{1}{N} \sum_{i=1}^{N}\left(T_{i}-P_{i}\right), \\
\sigma=\sqrt{\frac{\sum_{i=1}^{N}\left[\left(T_{i}-P_{i}\right)-\overline{\Delta T}\right]^{2}}{N-1},}
\end{gathered}
$$

where $N$ is the total number of items of equipment; $T_{i}$ is test data of the equipment $i ; P_{i}$ is predication of the equipment $i$.

\subsection{Temperature results}

In this study, the test data for steady-state condition 1 were

Table 1 Uncertain parameters

\begin{tabular}{ccc}
\hline Parameter description & Number of parameters & Maximum value \\
\hline Dry contact heat transfer coefficient $\left(\mathrm{W} \mathrm{m}^{-2} \mathrm{~K}^{-1}\right)$ & 2 & 50 \\
Contact heat transfer coefficient with thermal grease $\left(\mathrm{W} \mathrm{m}^{-2} \mathrm{~K}^{-1}\right)$ & 22 & 400 \\
Effective emissivity across MLI & 6 & 0.01 \\
Emissivity of polyimide film coating & 1 & 0.67 \\
Emissivity of S781 coating & 1 & 0.05 \\
\hline
\end{tabular}

Table 2 Conditions for calculation

\begin{tabular}{cccc}
\hline Conditions & \multirow{2}{*}{ Space external heat flux } & \multicolumn{2}{c}{ Active temperature control } \\
\cline { 3 - 5 } & & Batteries & Other equipment \\
\hline Steady-state condition 1 & maximum beta angle & $8^{\circ} \mathrm{C} \pm 0.5^{\circ} \mathrm{C}$ & $25^{\circ} \mathrm{C} \pm 0.5^{\circ} \mathrm{C}$ \\
Transient condition & summer solstice & $10^{\circ} \mathrm{C} \pm 0.5^{\circ} \mathrm{C}$ & $23^{\circ} \mathrm{C} \pm 0.5^{\circ} \mathrm{C}$ \\
Steady-state condition 2 & maximum beta angle & $10^{\circ} \mathrm{C} \pm 0.5^{\circ} \mathrm{C}$ & $23^{\circ} \mathrm{C} \pm 0.5^{\circ} \mathrm{C}$ \\
\hline
\end{tabular}
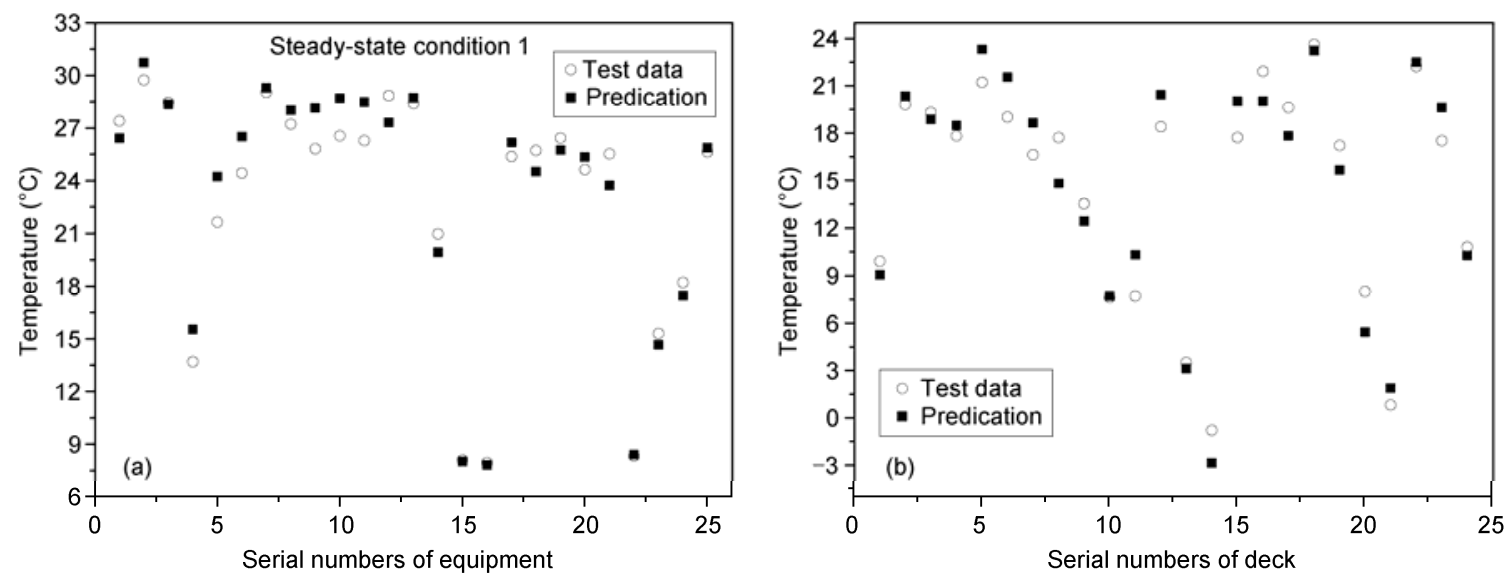

Figure 2 Comparison of test and calculated results in steady-state condition 1. (a) Equipment; (b) deck measuring points. 

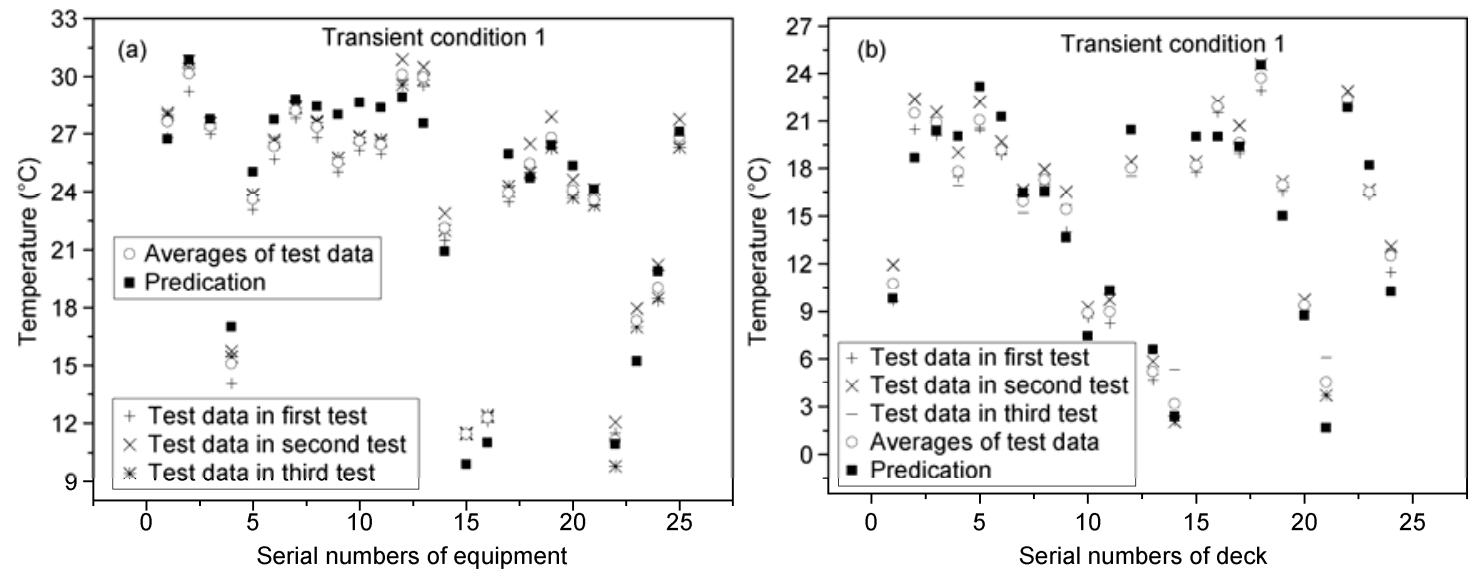

Figure 3 Comparison of test and calculated results in transient condition. (a) Equipment; (b) deck measuring points.

used to correct the thermal model, while the Transient Condition and Steady-state Condition 2 verified the corrected model [1]. Test and calculated temperatures of 25 items of equipment and 24 deck measuring points were compared. The results are shown in Figures 2-4. It can be seen that:

(1) After correction of the thermal model in Steady-state Condition 1, the average deviation between calculated data and test data is $-0.21^{\circ} \mathrm{C}$, and standard deviation $1.51^{\circ} \mathrm{C}$. All temperature deviations are within $\pm 3^{\circ} \mathrm{C}, 59$ and 70 percent of which are less than $\pm 1^{\circ} \mathrm{C}$ and $\pm 2^{\circ} \mathrm{C}$, respectively.

(2) Thermal analysis for the corrected model was done in the Transient Condition and Steady-state Condition 2. In the two conditions, the average deviations are $-0.08^{\circ} \mathrm{C}$ and $-0.56^{\circ} \mathrm{C}$, and the standard deviations are $1.56^{\circ} \mathrm{C}$ and $1.54^{\circ} \mathrm{C}$, respectively. All temperature deviations are less than $\pm 3^{\circ} \mathrm{C}$, of which less than $\pm 1{ }^{\circ} \mathrm{C}$ and $\pm 2{ }^{\circ} \mathrm{C}$ account for 55 and 76 percent in the Transient Condition, and 55 and 69 percent in the Steady-state Condition 2, respectively. These results suggest that the corrected thermal model is reasonably valid.

\subsection{Values and distribution of uncertain parameters}

The parameter values of the corrected thermal model are as follows. The dry contact heat transfer coefficients are 68.5 and $65.6 \mathrm{~W} \mathrm{~m}^{-2} \mathrm{~K}^{-1}$, respectively. The emissivities of the polyimide film coating and S781 coating are 0.69 and 0.89, respectively. There are 23 contact heat transfer coefficients with thermal grease and 6 effective emissivities across the MLI, shown in Figure 5(a) and (b) respectively.

As shown in Figure 5, it can be clearly seen that ranges of thermal contact heat transfer coefficient and effective emissivity across the MLI are $800-1600 \mathrm{~W} \mathrm{~m}^{-2} \mathrm{~K}^{-1}$ and $0.0103-0.0251$, averaging $1226.8 \mathrm{~W} \mathrm{~m}^{-2} \mathrm{~K}^{-1}$ and 0.0148 respectively. However, it is difficult to give the distributions of the two parameters. There are three main reasons: (1) the contact heat transfer coefficient is relevant to a variety of factors; (2) as a complex combination, there are many factors affecting the thermal performance of the MLI; (3) only limited statistical data of uncertain parameters were obtained in this study.
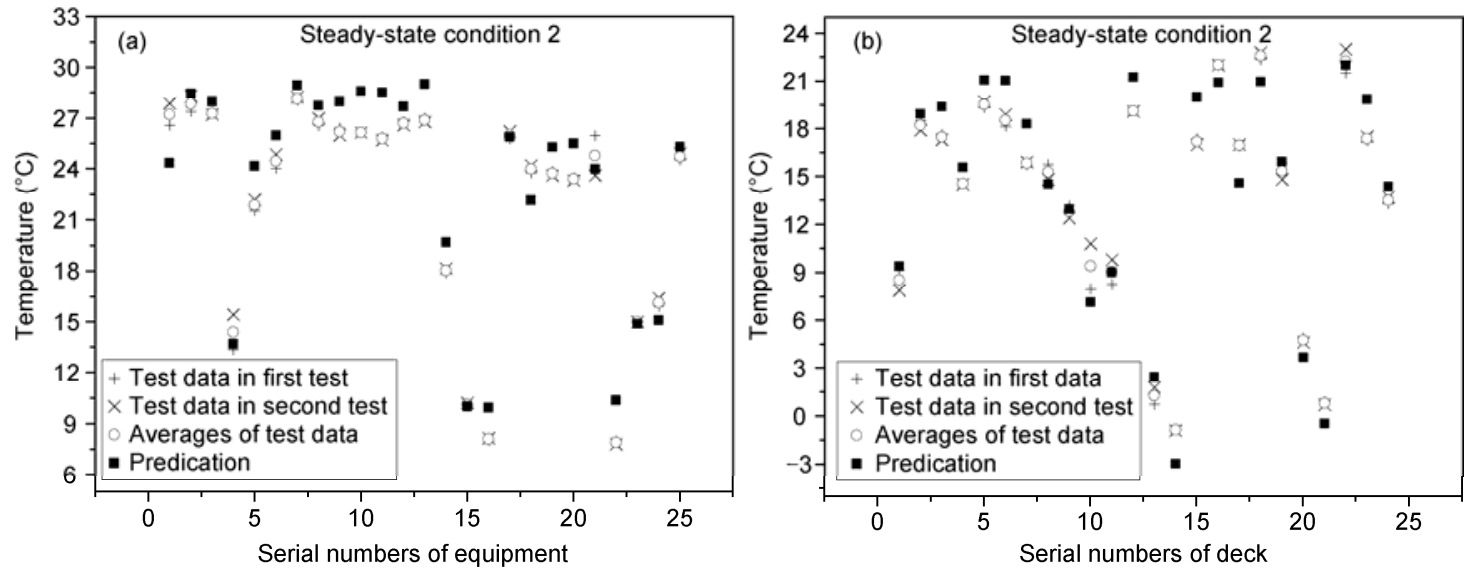

Figure 4 Comparison of test and calculated results in steady-state condition 2. (a) Equipment; (b) deck measuring points. 

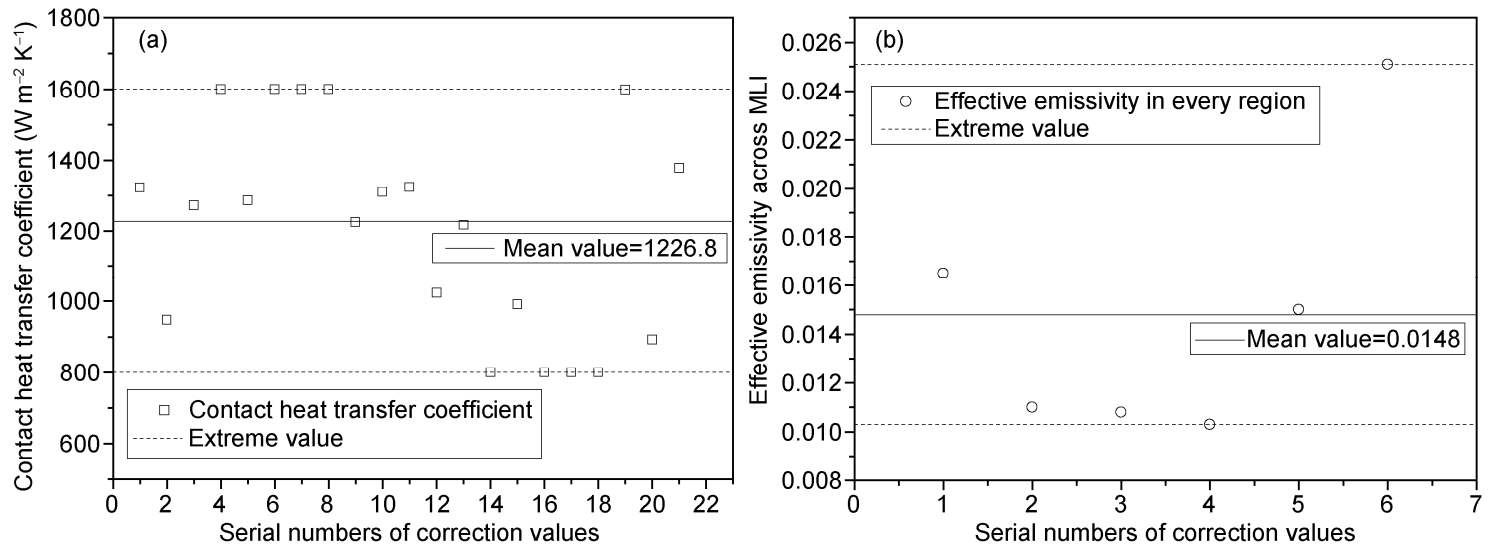

Figure 5 Parameter correction values. (a) Contact heat transfer coefficient; (b) effective emissivity across MLI.

\section{Conclusions}

In this study, the thermal model for a thermally controlled satellite in ground test conditions was corrected based on the Monte Carlo hybrid algorithm. The deviations between calculated and test data are all within $\pm 3^{\circ} \mathrm{C}$. At the same time, the parameter corrected values were given and ranges of contact heat transfer coefficient and effective emissivity across the MLI were also obtained. On the premise of accurate parameter sensitivity analyses, this method has a fast calculation speed and a relatively simple correction process. Overall, it is successful in engineering applications.

1 Hou Z Q, Hu J G. Spacecraft Thermal Control Technology (in Chinese). Beijing: China Science Technology Publishing House, 2007. $358,360,411-418$

2 Weng J H, Pan Z F. A Correction method for spacecraft thermal network and its coefficients (in Chinese). Chin Space Sci Technol, 1995, 15: $10-15$

3 Liu W, Jia $\mathrm{H}$. Thermal model correction for resources-2 satellite (in Chinese). Spacecraft Engin, 2003, 12: 29-35

4 Cruse T A, Wu Y T, et al. Probabilistic structural analysis methods and applications. Computers Structures, 1988, 30: 163-170

5 Pei L C, Wang Z Q. Monte Carlo Method and Its Application (in Chinese). Beijing: Beijing Ocean Press, 1988. 50-54
6 Wang J Y. Geophysical Inversion Theory (in Chinese). Beijing: Higher Education Press, 2002. 136-139

7 Cheng W L, Zhan H Z, Zhao R. A new method of thermal probe for thermal conductivities measurement based on Monte Carlo inversion (in Chinese). J China Univ Sci Tech, 2008, 38: 414-418

8 Wang Y M, Wang Y J, Wang W H, et al. FY-3 satellite ultraviolet total ozone unite. Chinese Sci Bull, 2009, 54: 3778-3783

9 Huang F X, Liu N Q, Zhao M X, et al. Vertical ozone profiles deduced from measurement of SBUS on FY-3 satellite. Chinese Sci Bull, 2009,54: 2556-2561

10 Zhang X M, Sun R M, Teng J W. Study on thickness of crust, lithosphere and asthenosphere in Qinghai-Tibet Plateau and its adjacent areas. Chinese Sci Bull, 2007, 52: 332-338

11 Herrera F L. Stochastic Approach to spacecraft thermal control subsystem. SAE 2000-01-2484 [2000, 7]. http://www.sae.org/technical/papers/2 000-01-2484

12 Yang H N, Zhong Q. Monte-Carlo method for thermal model correction of spacecraft (in Chinese). Spacecraft Engin, 2009, 18:53-58

13 Mareschi V, Perotto V, Gorlani M. Thermal test correlation with stochastic technique. SAE 2005-01-2855 [2005, 7]. http://www.sae.org/ technical/papers/2005-01-2855

14 Liu N, Cheng W L, Zhong Q, Fan Q M. Sensitivity analysis of spacecraft thermal model based on Monte-Carlo method (in Chinese). Spacecraft Engin, 2009, 18: 102-107

15 Cheng W L, Liu N, Zhong Q, Fan Q M. Study on parameters correction method of steady-state thermal model for spacecraft (in Chinese). J Astronautics, 2010, 1: 270-275

16 Ma H T, Hua C S. Research of thermal modeling method and temperature prediction for communication satellite platform. Chinese Space Sci Tech, 2002, 22: 54-60

17 Wan Z P, Fei P S. Optimization Theory and Method (in Chinese). Wuhan: Wuhan University Press, 2004. 62-76

Open Access This article is distributed under the terms of the Creative Commons Attribution License which permits any use, distribution, and reproduction in any medium, provided the original author(s) and source are credited. 